\title{
Functional genetic variants of CTNNBIPI predict platinum treatment response of Chinese epithelial ovarian cancer patients
}

\author{
Haoran Li ${ }^{1,2 \#, ~ L i h u a ~ C h e n ~}{ }^{1,2 \#, ~ X i a o x i a ~ T o n g 1,2, ~ H o n g j i ~ D a i ~}{ }^{3,4}$, Tingyan Shi ${ }^{5}$, Xi Cheng, ${ }^{2,6}$, Menghong Sun , \\ Kexin Chen ${ }^{3,4}$, Qingyi Wei ${ }^{1,8,9 \varpi}$ and Mengyun Wang ${ }^{1,2 \bowtie}$ \\ 1. Cancer Institute, Fudan University Shanghai Cancer Center, Shanghai, China. \\ 2. Department of Oncology, Shanghai Medical College, Fudan University, Shanghai, China. \\ 3. Department of Epidemiology and Biostatistics, Key Laboratory of Cancer Prevention and Therapy, Tianjin, China. \\ 4. Key Laboratory of Breast Cancer Prevention and Therapy, Ministry of Education, National Clinical Research Center for Cancer, Tianjin Medical University \\ Cancer Institute and Hospital, Tianjin, China. \\ 5. Ovarian Cancer Program, Division of Gynecologic Oncology, Department of Gynecology and Obstetrics, Fudan University Zhongshan Hospital, Shanghai, \\ China. \\ 6. Department of Gynecological Oncology, Fudan University Shanghai Cancer Center, Shanghai, China \\ 7. Department of Pathology, Tissue Bank, Fudan University Shanghai Cancer Center, Shanghai, China. \\ 8. Duke Cancer Institute, Duke University Medical Center, Durham, NC, USA. \\ 9. Department of Population Health Sciences, Duke University School of Medicine, Durham, NC, USA.
}

\#These authors contributed equally to this work.

$\square$ Corresponding authors: Mengyun Wang, Cancer Institute, Fudan University Shanghai Cancer Center, 270 Dong An Road, Shanghai, 200032, China; Tel: 86-21-64175590; E-mail: wangmengyun@fudan.edu.cn; and Qingyi Wei, Cancer Institute, Fudan University Shanghai Cancer Center, Shanghai, 200032, China; Tel: 86-21-64175590 E-mail address: weiqingyi@fudan.edu.cn; or Duke Cancer Institute, Duke University Medical Center, Durham, North Carolina, 27710, USA. Tel: 919-660-0562; E-mail address: qingyi.wei@duke.edu. Qingyi Wei is a visiting professor at Fudan University.

(c) The author(s). This is an open access article distributed under the terms of the Creative Commons Attribution License (https://creativecommons.org/licenses/by/4.0/). See http://ivyspring.com/terms for full terms and conditions.

Received: 2020.05.15; Accepted: 2020.09.15; Published: 2020.09.30

\begin{abstract}
Chemotherapy resistance remains a blockade for successful treatment and longer overall survival of patients with epithelial ovarian cancer (EOC). CTNNBIPI is an inhibitor of $\beta$-catenin that is a chemotherapeutic target for EOC treatment. In the present study, we investigated associations between single nucleotide polymorphisms (SNPs) of CTNNBIPI and platinum treatment response of Han Chinese EOC patients and subsequently performed functional prediction and validation of the resultant SNPs. We found that CTNNBIPI rs935072 AT/TT variant genotypes were associated with platinum treatment response in the multivariate logistic regression analysis of EOC patients. Specifically, the CTNNBIPI rs935072 AT/TT genotypes were associated with a decreased risk of developing chemoresistance ([adjusted odds ratio $(\mathrm{OR})]=0.89,95 \%$ confidence interval $(\mathrm{Cl})=0.82-0.97$ and $P=0.010$ ), compared with the AA genotype. Further experiments showed that the underlying mechanism for the CTNNBIPI rs935072 A>T change in chemotherapy treatment response resulted from a lower binding affinity of miR-27a-3p, thereby leading to up-regulation of the CTNNBIPI expression. We further found that overexpression of CTNNBIPI sensitized ovarian cancer cells to platinum treatment. Thus, the present study provides evidence that functional variants of CTNNBIPI may regulate the expression of CTNNBIPI, a possible mechanism affecting platinum treatment response of EOC patients.
\end{abstract}

Key words: genetic variants, single nucleotide polymorphisms; ovarian cancer; CTNNBIP1; platinum treatment response

\section{Introduction}

Chemotherapeutic drug resistance in tumor cells is presented as a major cause of significant mortality in human malignancies [1,2], especially in ovarian cancer [3]. Currently, the first-line chemotherapy treatment for ovarian cancer is the paclitaxel-platinum combination, which is given every three weeks for six 
or eight cycles. The platinum-based regimen yields an objective response rate of $>80 \%$ (in which $40-60 \%$ of the patients had a complete response rate) in the advanced ovarian cancer, but approximately $80 \%$ of the patients with an advanced stage had a progression within three years [4] with a median progression-free survival of only about 18 months, which is mainly attributed to the development of chemoresistancerelated recurrence [3]. Furthermore, most women diagnosed with epithelial ovarian cancer (EOC) develop an acquired chemoresistance in spite of an initial response to the treatment. As a result, chemotherapy resistance remains a blockade for successful treatment and a longer overall survival (OS) in EOC patients [5]. Therefore, to have a better management of EOC, it is essential to elucidate the mechanisms of chemoresistance and to identify potential biomarkers to predict chemotherapy response among EOC patients.

It is known that genetic variants are involved in tumor development and patients' survival from drug treatment [6] and thereby considered to participate in the resistance to the anti-cancer drugs [7, 8]. For example, genetic variation in biological signaling pathways, including drug uptake and efflux mechanisms, cell cycle, DNA damage repair, apoptosis and glucose metabolism pathways, has been shown to be involved in different treatment responses to chemotherapy in several cancer types [9-11], including EOC [12, 13]. However, the underlying genetic mechanisms responsible for such chemotherapy resistance in EOC patients are still poorly understood.

Susceptibility alleles identified from genomewide association studies (GWASs) easily omit the truly biologically significant ones because of the strict $p$-value standard, so the candidate gene association studies have also been used as a supplementary method to find the missed alleles. Recently, the Wnt/ $\beta$-catenin pathway was reported to be involved in the chemoresistance of EOC patients, which may be a potential target for chemosensitization [14]. Catenin beta interacting protein 1 (CTNNBIP1), also known as ICAT or an inhibitor of $\beta$-catenin, prevents $\beta$-catenin from forming a complex with the T-cell factor/ lymphoid enhancer factor (TCF/LEF) and thus inactivates the transcription of Wnt target genes, thereby negatively regulating the $W n t / \beta$-catenin pathway [15]. Furthermore, several studies have suggested CTNNBIP1 as a tumor suppressor in cancers of the colorectum [16], breasts [17], cervix [18] and stomach [19]. Therefore, these findings provide some clues that CTNNBIP1 may be a chemotherapeutic target in these cancers.

However, few studies explored the role of
CTNNBIP1 in EOC treatment, and, in particular, single nucleotide polymorphisms (SNPs) of CTNNBIP1 have rarely been investigated for their roles in the response to platinum treatment of EOC patients. Therefore, we hypothesize that functional genetic variants in CTNNBIP1 are associated with the response to platinum treatment of EOC patients. In the present study, we first used available genotyping data of CTNNBIP1 SNPs for association analysis and then performed validation of the significant SNPs identified in the multivariate logistic regression analysis, followed by functional analysis of these SNPs.

\section{Materials and Methods}

\section{Study population}

All the patients included in the present study were unrelated ethnic Han Chinese women diagnosed with histologically confirmed EOC, and their DNA samples were obtained from the blood samples collected by the tissue bank of Fudan University Shanghai Cancer Center (FUSCC). The present study included a total of 495 EOC patients consecutively enrolled for Shanghai Ovarian Cancer Study (SOCS) at FUSCC with complete DNA samples between March 2009 and August 2012. Because the present study mainly focused on associations between SNPs and the response to platinum treatment of EOC patients, we excluded those patients who had not undergone any platinum-based chemotherapies. Therefore, 427 patients were left in the final dataset, whose participation in the present study was approved by the Ethics Committee at FUSCC with a written-informed consent obtained from all recruited individuals. This investigation was conducted according to the principles in the Declaration of Helsinki consent.

\section{Data collection}

Clinical characteristics, including age at diagnosis, the International Federation of Gynecology and Obstetrics (FIGO) stage, histology, grade, residue (optimal debulking $<1 \mathrm{~cm}$ ), ascites and platinum treatment response were collected. Primary patients with an early stage (FIGO stage I and II) received a complete staging surgery, while patients with a late stage (FIGO stage III and IV) underwent a cytoreductive surgery. After a primary surgery, all the patients received platinum-based chemotherapy. Those patients who received chemotherapy were divided into a chemosensitive group (relapsed $>6$ months after chemotherapy) and a chemoresistant group (relapsed $\leq 6$ months after chemotherapy). All the patients were followed up every three months for 
the first two years, every 6 months for the next three years, and annually for the following years thereafter.

\section{Genotyping data and quality control}

The genotyped data were obtained from a previous GWAS study that used the Illumina HumanOmni Zhonghua-8 BeadChip for genotyping as described previously [20], and we only selected CTNNBIP1 as the candidate gene for the analysis. Systematic quality control was performed and the exclusion criterion were as follows: 1) a call rate less than $95 \%$; 2) mapping to $X$ or $Y$ chromosomes; 3 ) a minor allele frequency <0.05; and 4) with HardyWeinberg equilibrium $P<1 \times 10^{-5}$. In addition, we analyzed the principle component and found no significant principle component to be related with the response to platinum treatment of EOC patients.

\section{Imputation}

Additional SNPs of CTNNBIP1 not on the GWAS chips were imputed using IMPUTE 2.0 (https://mathgen.stats.ox.ac.uk/impute/impute_v2. html). The reference data used for imputation was from the 1000 Genomes Project (phase 3). The imputed genotypes were qualified by excluding SNPs with the following criteria: a posterior probability < 0.9 , minor allele frequency $<5 \%$, missing genotypes $>10 \%$ or significant deviations from Hardy-Weinberg equilibrium.

\section{False-positive report probability and Bayesian false-discovery probability}

False Discovery rate (FDR), false-positive report probability (FPRP), and Bayesian false-discovery probability (BFDP) were used to assess the noteworthiness of an observed association between genetic variants and platinum response. The detailed calculation method of FDR [21], BFDP [22] and FPRP [23] were described previously. The stringent FDR method was not optimal for the SNPs under investigation in the present study, because they are in high linkage disequilibrium (LD) as a result of imputation, a threshold of FPRP value less than 0.2 and BFDP value less than 0.8 were considered statistically noteworthy.

\section{Association analysis}

To screen of relevant SNPs associated with platinum response, we used Package GenABEL [24] in $\mathrm{R}$ language to perform logistic regression analyses, with correction for co-variables including age, stage, histology, grade, residue, ascites and neoadjuvant chemotherapy. The LD analysis among the obtained SNPs were performed by using Haploview [25]. Receiver operating characteristic (ROC) curve was used to estimate the predictive value of genetic variants in combination with clinical variables in additive models. To illustrate the fitness of the model, an area under the curve (AUC) of ROC curves was also calculated.

\section{Functional annotation of selected SNPs}

The online tools RegulomeDB (http://www. regulomedb.org/), SNPInfo (https://snpinfo.niehs. nih.gov/) and Ensemble (http://www.ensembl.org/) were applied to predict putative function of the identified SNPs. Furthermore, the corresponding mRNA expression levels of the identified loci was assessed by using the online tool GTEx database (http://www. gtexportal. org/ home/)_[26] as well as data from the 1000 Genomes Project and from the Hapmap3 (phase III, release I) Project. We also used other online tools including MirSNP (http:// bioinfo.bjmu.edu.cn/mirsnp/search/), TargetScan (http://www.targetscan.org/vert_71/) and SNPInfo (https://snpinfo.niehs.nih.gov/) to predict potential miRNA binding with the 3'UTR region of the identified loci. The online tools GEPIA (http:// gepia.cancer-pku.cn) and Oncomine Database (https://www.oncomine.org/) were also used to explore gene expression levels in different tissues.

\section{Cell lines and culture}

Two established human ovarian cancer cell lines (i.e., IGROV1 and OVCAR-8) were obtained from the Cell Bank of the Eastern China in December 2016. The identities of cell lines were confirmed by DNA profiling (short tandem repeat, STR). All cell lines were used within 6 months after receipt or resuscitation. All cells were cultured in Dulbecco's modified Eagle's medium (DMEM, HyClone, Thermo Scientific, USA) supplemented with $10 \%$ fetal bovine serum (Gibco, Life technologies, USA), $100 \mathrm{U} / \mathrm{ml}$ penicillin (Biowest, Nuaillé, France), and $100 \mathrm{U} / \mathrm{ml}$ streptoc-mycin (Biowest, Nuaillé, France), and were incubated at $37^{\circ} \mathrm{C}$ in a humidified atmosphere with $5 \% \mathrm{CO}_{2}$.

\section{Cell transfection}

To selectively overexpress CTNNBIP1, the recombinant plasmid pENTER-CTNNBIP1 containing human full cDNA sequence of CTNNBIP1 was purchased from Vigene Biosciences (Jinan, China). Both ovarian cancer cell lines IGROV1 and OVCAR-8 were infected with the recombinant plasmids. Control cell lines were generated by infection with plasmids containing the empty vector with the same experimental protocol. To generate miR-27a-3p overexpressing cells, miR-27a-3p mimic or their negative control (Biotend, Shanghai, China) was transfected into cells using Lipofectamine 3000 (Life 
Technologies, Carlsbad, CA) according to the manufacturer's instructions.

\section{Luciferase reporter assay}

The Psi-CHECK2 vector carrying the 3'UTR of CTNNBIP1 with either rs935072 A or rs935072 T was constructed. IGROV1 and OVCAR-8 cells were transfected with an appropriate dose of constructed test plasmids and renilla luciferase control plasmid in 96-well plates. Forty-eight hours later, luciferase activities were measured by using the Dual Luciferase Assay Kit (Promega, Madison, WI, USA), and renilla luciferase activities were used to normalize the reporter luciferase activities, which were then rescaled to vector control signals equal to unit 1 .

\section{Cell viability assay}

To evaluate cell survival fraction under different cisplatin concentrations, we plated $8 \times 10^{3}$ cells per well in 96-well plates with $100-\mu l$ maintenance medium. On the next day, the cells were treated with various concentrations of cisplatin. Cell Counting Kit- 8 (Dojindo Laboratories, Kumamoto, Japan) was used to monitor cell viability 48 hours later, and the number of viable cells was assessed by measurement of absorbance at $450 \mathrm{~nm}$ by a Microplate Reader (BioTek Instruments, Winooski, VT, USA).

\section{Colony formation assay}

Colony formation Assay was performed as previously described [27].

\section{Cell apoptosis analysis}

Cell apoptosis analysis was performed as previously described [27].

\section{Reverse transcription quantitative real-time polymerase chain reaction}

Total RNAs were isolated from both IGROV1 and OVCAR- 8 cells by using the Trizol reagent (Invitrogen, Life technologies, USA) and reversely transcribed into cDNA using the PrimeScript TM RT reagent Kit (Takara Biotechnology, Shiga, Japan). The primer pairs of GAPDH were 5'-GGCCTC CAAGGA GTAAGACC-3' (forward primer) and 5'-CAAGGGG TCTACATGGCAAC-3' (reverse primer). The primer pairs of CTNNBIP1 were 5'-GGGCGGCACCTT CCT-3' (forward primer) and 5'-CTCTGGGGACTCC TGCTTCT-3' (reverse primer). Three independent experiments were performed for final analyses by using the $2^{-\Delta \Delta C T}$ relative quantification method.

\section{Western blotting assay}

Antibodies against CTNNBIP1 were purchased from Proteintech (Wuhan, Hubei). All the primary antibodies were used at 1:1000 dilutions and secondary antibodies at 1:5000 dilutions. The assay was performed three times as previously described [27].

\section{Statistical analysis}

Genotyping data were extracted by PLink (version 1.09) (http://pngu.mgh.harvard.edu/ purcell/plink/) [28]. Other statistical analyses were achieved by $\mathrm{R}$ language (version 3.2.4). $\chi^{2}$ test and logistic regression models were used to estimate the main effects of the SNPs on platinum treatment response. The heterogeneity between two groups was assessed by the Cochran's Q-test. The figures were made using GraphPad Prism and phototshop expressed as mean and standard deviation (SD). Statistical analysis was conducted by Student's $t$-test. All the reported $P$ values were two-sided, and $P<0.05$ was considered statistically significant.

\section{Results}

\section{Logistic regression analysis of associations between SNPs and platinum treatment response}

The study flowchart is shown in Figure 1. The CTNNBIP1 genotyping data (nine SNPs) of 427 patients who underwent platinum-based treatment were available for subsequent analysis. After quality control and imputation, 93 SNPs in CTNNBIP1 were included for further analysis, of which nine SNPs were genotyped and 84 were imputed. As a result, we found that 49 SNPs in CTNNBIP1 were individually and significantly associated with the response to platinum treatment of EOC patients (adjusted $P<0.05)$. To control for the probability of false positive associations with platinum treatment response of EOC patients, both FPRP and BFDP were performed and 11 SNPs passed the corrections (Table S1), although false discovery rate (FDR) was not applied because of the high LD among the SNPs as a result of imputation. The following LD analysis revealed that these 11 SNPs were in LD $\left(r^{2}>0.8\right)$, one of which, CTNNBIP1 rs935072A>T, is located at the 3'- UTR of the gene and was selected for further genetic modeling and functional validation. CTNNBIP1 rs935072A $>\mathrm{T}$ was found to be associated with platinum treatment response ([adjusted odds ratio $(\mathrm{OR})]=0.62,95 \%$ confidence interval $(\mathrm{CI})=0.44-0.88$, $P=0.009$ and $\mathrm{FPRP}=0.164, \mathrm{BFDP}=0.568)$. Furthermore, we performed the stepwise multivariate logistic regression analysis to select the optimal predictors of platinum treatment response in EOC patients, with adjustment for other clinical variables including age, FIGO stage, histology, grade, residue (optimal debulking $<1 \mathrm{~cm}$ ), ascites and neoadjuvant 
chemotherapy. The above-mentioned CTNNBIP1 rs935072 SNP still remained noteworthy (Table S2). In addition to age (adjusted OR=1.08, 95\% $\mathrm{CI}=0.99-1.18, P=0.057$ ), FIGO stage (adjusted $\mathrm{OR}=1.15$, 95\% $\quad \mathrm{CI}=1.03-1.29, \quad P=0.015), \quad$ residue (adjusted OR=1.31, 95\% CI=1.15-1.49, $P<0.001)$, the CTNNBIP1 rs935072A $>\mathrm{T}$ remained an independent predictive factor (adjusted OR=0.92, 95\% CI=0.87-0.98, $P=0.014$ ) of platinum treatment response in EOC patients.

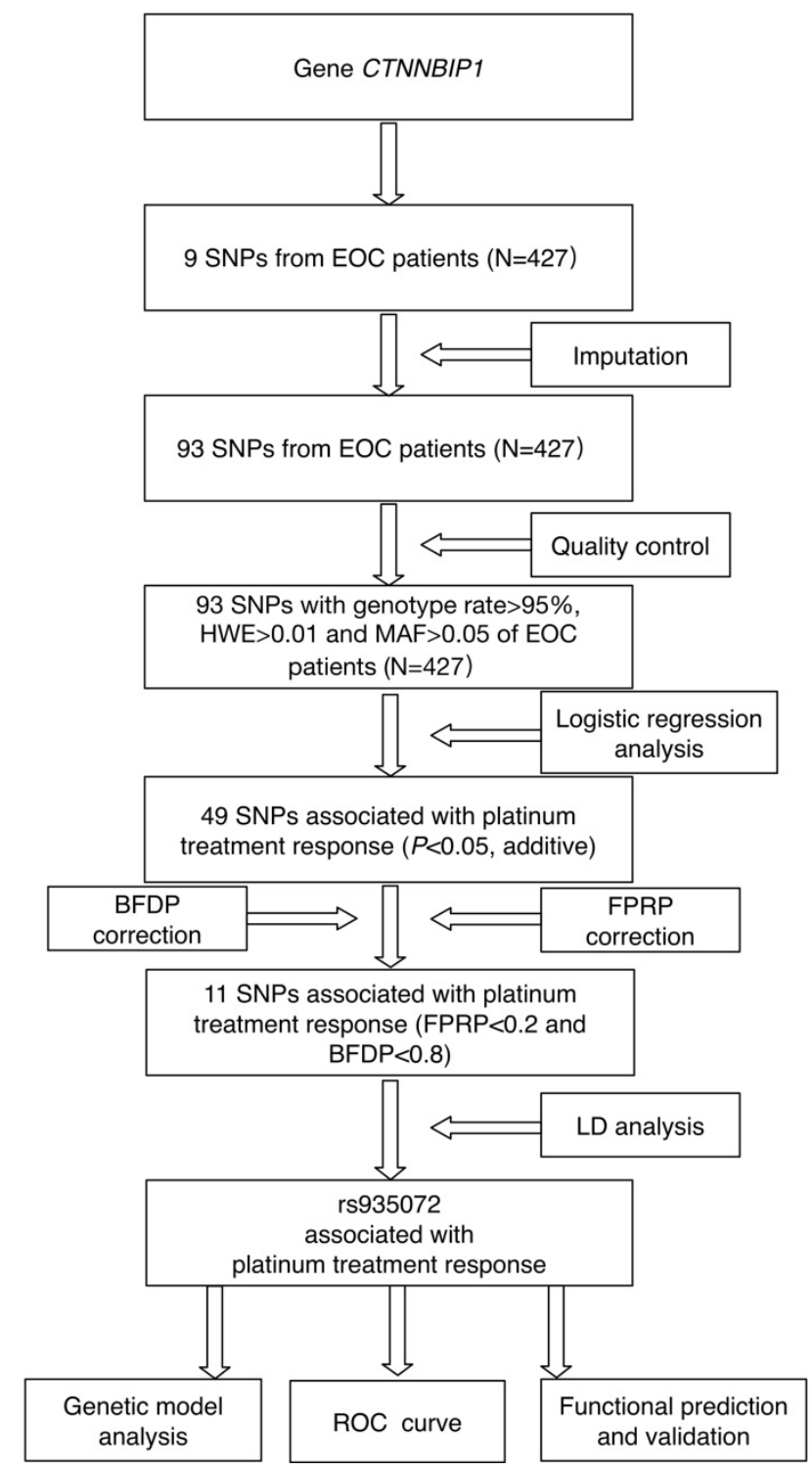

Figure 1. The study analysis flowchart.

\section{Genetic association with platinum treatment response of EOC patients}

The genotype (A/A, A/T, and $\mathrm{T} / \mathrm{T})$ frequencies of the CTNNBIP1 rs935072 SNP were $50.6 \%, 39.6 \%$, and $9.8 \%$, respectively, and the allele $\mathrm{A}$ and $\mathrm{T}$ frequencies were $70.4 \%$ and $29.6 \%$, respectively. In the multivariate analysis with adjustment for age, FIGO stage, histology, grade, residue (optimal debulking $<1$ $\mathrm{cm})$, ascites and neoadjuvant chemotherapy, the CTNNBIP1 rs935072 AT/TT genotypes were associated with a decreased risk of developing chemoresistance (adjusted OR=0.89, 95\% CI=0.82-0.97 and $P=0.010$ ), compared with the AA genotype (Table 1). Then, we used diagnostic ROC curve to assess discriminative accuracy of the identified SNP CTNNBIP1 rs935072A $>$ T. First, we constructed a logistic regression model with the above-mentioned clinical variables. Then, we added rs935072 to the model in an additive genetic model. Although the ROC prediction model incorporating CTNNBIP1 rs935072 was borderline significantly different from that incorporating only clinical factors, the AUC was increased (all patients: AUC 0.671 vs. 0.692, 95\% $\mathrm{CI}=0.615-0.726$ vs. $0.638-0.747, P=0.057$, Figure S2).

\section{Stratified analysis between unfavorable genotypes and platinum treatment response}

Then, we performed stratified analysis to select the subgroup of patients who could benefit more from the identified SNP in the combination with other predictors (Table 2), including age, tumor grade, histology, FIGO stage, residue, ascites as well as neoadjuvant chemotherapy. We found that CTNNBIP1 rs935072A > T was more effective to predict the platinum response in subgroups of patients who were older than 54 (adjusted OR=0.83, 95\% $\mathrm{CI}=0.73-0.95, P=0.006$ ), with a high grade (adjusted $\mathrm{OR}=0.90,95 \% \mathrm{CI}=0.83-0.99, P=0.022$ ) or serous tumors (adjusted OR=0.83, 95\% CI=0.72-0.97, $P=0.020$ ), with an advanced tumor stage (adjusted OR=0.88, 95\% $\mathrm{CI}=0.80-0.98, \quad P=0.017)$, and without neoadjuvant chemotherapy (adjusted OR $=0.87,95 \%$ CI $=0.80-0.96$, $P=0.005)$. No interactive effects between covariates and platinum treatment responseassociated genotypes were identified, except for an interaction between CTNNBIP1 rs935072 and tumor grade $(P=0.037)$.

\section{Functional prediction of CTNNBIPI rs935072A>T}

To investigate the role of CTNNBIP1 rs935072A $>\mathrm{T}$ in regulating its gene expression, we searched the GTEx database where both mRNA expression and genotype data were available. Initial analysis found that the mRNA expression levels were up-regulated by the CTNNBIP1 rs935072 A>T change in whole blood cells $(P=0.023$, Figure 2A). Despite no statistical change in ovary tissues, patients $(n=38)$ with the AT genotype showed an increased CTNNBIP1 mRNA expression level compared with those $(n=75)$ with the AA genotype $(P=0.570$, Figure 2B). We also performed the expression quantitative 
trait loci analysis for the correlation between the SNP and mRNA expression levels by using data in the 1000 Genomes Project ( $\mathrm{n}=373$ and $P=0.937$, Figure S3A) and the Hapmap3 project of Chinese subjects $(n=79$ and $P=0.671$, Figure S3B). Unfortunately, no significant difference in the expression levels was observed by the $\mathrm{A}>\mathrm{T}$ change due to the relative small number of patients.

We further searched in the TCGA database for the mRNA expression levels of CTNNBIP1 in both EOC and normal ovarian tissues, but there was no difference in CTNNBIP1 mRNA expression levels between EOC and normal ovarian tissues. However, we found that CTNNBIP1 mRNA expression was down-regulated as the stage increased (Figure 2C), indicating its role in tumor progression. In addition, because ovarian serous carcinoma is the most sensitive type to common platinum-based chemotherapy than other types such as mucinous [29] or clear cell carcinoma [30], we then searched the Oncomine database to analyze mRNA expression levels of CTNNBIP1 by different histology subtypes of ovarian cancer. Strikingly, the mRNA expression levels of CTNNBIP1 was much higher $(P=0.009)$ in serous tumor type $(n=30)$ than in other types $(n=8)$ (Figure 2D). Taken together, the underlying protective mechanism of the rs935072 A>T may be attributed to altered mRNA expression levels of CTNNBIP1.
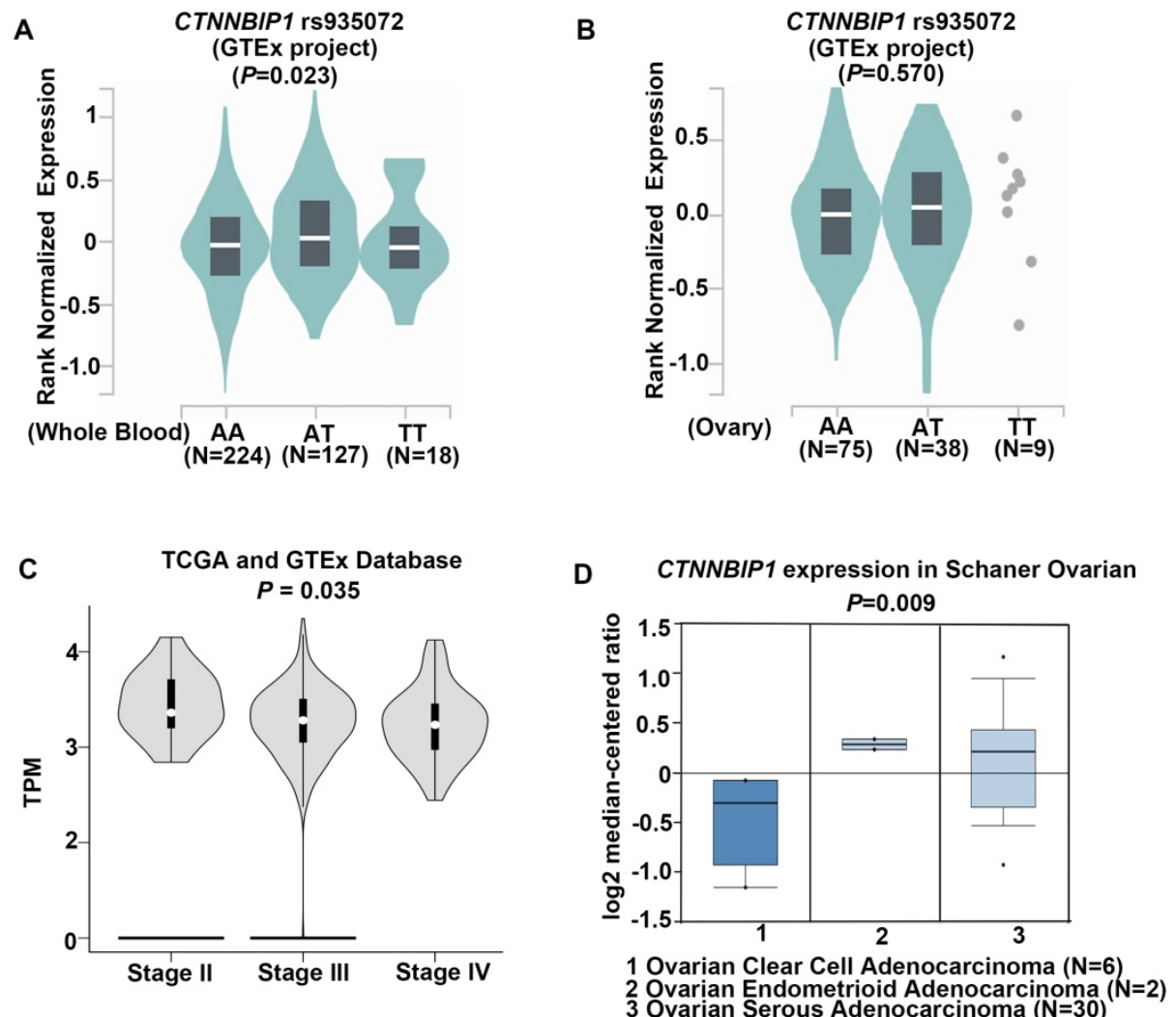

Figure 2. Functional prediction of genetic variants of CTNNBIPI. A, The impact of CTNNBIPI rs 935072 SNP on the mRNA expression of CTNNBIPI in whole blood tissues from the GTEx database. B, The impact of CTNNBIPI rs935072 SNP on the mRNA expression of CTNNBIPI in ovary tissues from the GTEx database. c, CTNNBIPI expression in different stage of ovarian cancer tissues from TCGA database. TPM, Transcripts per Kilobase of an exon model per Million mapped reads. C, CTNNBIPI expression in different histology of ovarian cancer tissues from Oncomine database.

Table 1. Association of CTNNBIPI rs935072 with platinum treatment response of EOC patients

\begin{tabular}{|c|c|c|c|c|c|c|}
\hline \multirow[t]{3}{*}{ Genetic variant } & \multirow[t]{3}{*}{ Genotype } & \multirow[t]{3}{*}{ Number/Event } & \multicolumn{4}{|c|}{ Platinum treatment response } \\
\hline & & & \multicolumn{2}{|l|}{ Univariate } & \multicolumn{2}{|l|}{ Multivariate } \\
\hline & & & OR $(95 \% \mathrm{CI})$ & $P$ & OR $(95 \% \mathrm{CI})$ & $P^{*}$ \\
\hline CTNNBIP1 & AA & $216 / 80$ & 1.00 & & 1.00 & \\
\hline \multirow[t]{2}{*}{ rs935072 A > T } & AT & $169 / 45$ & $0.90(0.82-0.99)$ & 0.029 & $0.90(0.82-0.98)$ & 0.022 \\
\hline & TT & $42 / 9$ & $0.86(0.73-0.99)$ & 0.046 & $0.87(0.75-1.02)$ & 0.079 \\
\hline Dominant model & $\mathrm{AA} / \mathrm{AT}+\mathrm{TT}$ & $211 / 54$ & $0.89(0.82-0.97)$ & 0.011 & $0.89(0.82-0.97)$ & 0.010 \\
\hline Recessive model & $\mathrm{AA}+\mathrm{AT} / \mathrm{TT}$ & $385 / 125$ & $0.90(0.77-1.04)$ & 0.144 & $0.92(0.79-1.06)$ & 0.234 \\
\hline Additive model & $\mathrm{AA} / \mathrm{AT} / \mathrm{TT}$ & & $0.92(0.86-0.98)$ & 0.010 & $0.92(0.86-0.98)$ & 0.013 \\
\hline
\end{tabular}

Abbreviations: EOC, epithelial ovarian carcinoma; OR, odds ratio; $\mathrm{CI}$, confidence interval;

$P^{*}$, the multivariate logistic regression analyses were adjusted for age, tumor grade, histological types, FIGO stage, residue, ascites and neoadjuvant chemotherapy;

The results were in bold if $P<0.05$ 
Table 2. Stratification analysis for associations between CTNNBIPI rs935072 A>T and platinum treatment response of the EOC patients

\begin{tabular}{|c|c|c|c|c|c|}
\hline \multirow[t]{2}{*}{ Variables } & \multicolumn{2}{|c|}{$\begin{array}{l}\text { CTNNBIP1 rs935072 } \\
\text { (Number/Event) }\end{array}$} & \multicolumn{3}{|c|}{ Platinum treatment response } \\
\hline & $\mathrm{AA}$ & AT \& TT & OR $(95 \% \mathrm{CI})$ & $P^{*}$ & $P^{\dagger}$ \\
\hline Age at diagnosis & & & & & 0.176 \\
\hline$<54$ & $116 / 34$ & $112 / 27$ & $0.94(0.84-1.06)$ & 0.326 & \\
\hline$\geq 54$ & $100 / 46$ & $99 / 27$ & $0.83(0.73-0.95)$ & 0.006 & \\
\hline Grade & & & & & 0.037 \\
\hline Low & $6 / 3$ & $7 / 0$ & $0.43(0.22-0.84)$ & 0.089 & \\
\hline High & $209 / 77$ & $204 / 54$ & $0.90(0.83-0.99)$ & 0.022 & \\
\hline $\begin{array}{l}\text { Histological } \\
\text { types }\end{array}$ & & & & & 0.278 \\
\hline Serous & $151 / 55$ & $143 / 40$ & $0.92(0.83-1.02)$ & 0.105 & \\
\hline Others $\ddagger$ & $65 / 25$ & $68 / 14$ & $0.83(0.72-0.97)$ & 0.020 & \\
\hline FIGO Stage & & & & & 0.620 \\
\hline I - II & $39 / 7$ & $35 / 5$ & $0.93(0.77-1.12)$ & 0.435 & \\
\hline III - IV & $170 / 71$ & $158 / 47$ & $0.88(0.80-0.98)$ & 0.017 & \\
\hline Residue disease & & & & & 1.000 \\
\hline$\leq 1 \mathrm{~cm}$ & $143 / 42$ & $133 / 28$ & $0.92(0.83-1.02)$ & 0.105 & \\
\hline$>1 \mathrm{~cm}$ & $28 / 17$ & $27 / 14$ & $0.92(0.83-1.02)$ & 0.105 & \\
\hline Ascites & & & & & 0.451 \\
\hline No & $33 / 9$ & $26 / 2$ & $0.85(0.70-1.04)$ & 0.129 & \\
\hline Yes & $130 / 50$ & $130 / 40$ & $0.93(0.83-1.04)$ & 0.202 & \\
\hline Neoadjuvant & & & & & 0.291 \\
\hline No & $175 / 67$ & $177 / 43$ & $0.87(0.80-0.96)$ & 0.005 & \\
\hline Yes & $41 / 13$ & $34 / 11$ & $0.99(0.80-1.23)$ & 0.922 & \\
\hline $\begin{array}{l}\text { Abbreviations: EC } \\
\text { interval; }\end{array}$ & epith & ovarian & oma; OR, odd & ; CI & dence \\
\hline $\begin{array}{l}P^{\star}-P \text { value of mul } \\
\text { grade, histological } \\
\text { chemotherapy; } \\
P^{\dagger}-P \text { value of Coc } \\
{ }^{\ddagger} \text { - other histologica } \\
\text { types of EOC; }\end{array}$ & $\begin{array}{l}\text { tivariate lo } \\
\text { types, FIC } \\
\text { hran's Q } t \\
\text { al types in }\end{array}$ & $\begin{array}{l}\text { gistic regress } \\
\text { est for hetero } \\
\text { clude mucino }\end{array}$ & $\begin{array}{l}\text { n analyses was ad } \\
\text { e, ascites and neo } \\
\text { neity between the } \\
\text {, endometrioid, } \mathrm{cl}\end{array}$ & $\begin{array}{l}\text { isted for } \\
\text { ljuvant } \\
\text { No grour } \\
\text { ar cell an }\end{array}$ & $\begin{array}{l}\text { ge, tumor } \\
\text {; } \\
\text { others }\end{array}$ \\
\hline
\end{tabular}

\section{The effect of CTNNBIPI rs935072 A>T on the binding ability of miR-27a-3p and 3'- UTR of CTNNBIPI}

Then, we explored putative function of CTNNBIP1 rs935072 located at the $3^{\prime} \mathrm{UTR}$, which is a critical binding site of microRNA. We hypothesized that the rs935072 $\mathrm{A}>\mathrm{T}$ change might reduce the binding capacity of miRNA, thereby affecting the expression of CTNNBIP1 and platinum treatment response of EOC patients. Therefore, we used the online tools of MirSNP (http://bioinfo.bjmu. edu.cn/mirsnp/search/), TargetScan (http://www. targetscan.org/vert_71/) and SNPInfo (https:// snpinfo.niehs.nih.gov/) to predict the specific microRNA binding with the CTNNBIP1 3'UTR. The results showed that miR-27a-3p was a potential microRNA that could bind to the 3'UTR of CTNNBIP1 (Figure 3A). Specifically, the CTNNBIP1 rs935072 A>T change may affect the binding of CTNNBIP1 3'UTR with miR-27a-3p. To test this hypothesis experimentally, we constructed a Psi-CHECK2 vector carrying the 3'UTR of CTNNBIP1 with rs935072 A or T allele (Figure 3B). The sequencing results of the Psi-CHECK2 vector containing either rs935072 A or T allele are shown in Figure 3C. In both IGROV1 and OVCAR-8 cell lines, when overexpressing miR-27a$3 p$, we observed a down-regulated luciferase activity associated with the rs935072 A allele in the luciferase reporter assay, and this effect was abrogated when the rs935072 A allele changed into the T allele (Figure 3D and $3 \mathrm{E})$. In addition, we found that the miR-27a-3p mimic could significantly reduce both mRNA and protein expression levels of CTNNBIP1 (Figure 3F-3G). Together with the results that rs935072 A>T change affected the efficiency of microRNA-27a-3p binding, we speculated that a weak binding affinity of miR-27a-3p to the $3^{\prime} \mathrm{UTR}$ region may result in the up-regulation of the CTNNBIP1 effectively.

\section{CTNNBIPI sensitizes ovarian cancer cells to cisplatin}

To further explore the effect of CTNNBIP1 on chemotherapy sensitivity of ovarian cancer, we treated IGROV1 and OVCAR-8 cell lines selectively overexpressing CTNNBIP1 and their corresponding control cells with different concentrations of cisplatin for $48 \mathrm{~h}$. The Cell Counting Kit- 8 assays (Figure 4A-4B) revealed that the overexpression of CTNNBIP1 increased the sensitivity to cisplatin. Colony formation assay results clearly showed that the overexpression of CTNNBIP1 reduced the number and size of the colonies after treatment with cisplatin (Figure 4C-4D). Then, we performed flow cytometry analysis to measure apoptotic cells 48 hours after treatment with cisplatin. Compared with control cells, the overexpression of CTNNBIP1 induced more cell death (Figure 4E) in all cell lines tested. Taken together, our experimental data suggested that CTNNBIP1-overexpressing cells had a higher rate of apoptosis than control cells in response to cisplatin treatment and that CTNNBIP1 over-expression synergized with cisplatin to inhibit cell proliferation.

\section{Discussion}

In the present study, we found that 11 out of the single-locus analysis of 93 CTNNBIP1 SNPs were associated with chemotherapy response. In the LD analysis, the CTNNBIP1 rs935072 A>T SNP captured other 10 SNPs in a high LD block. Further stepwise multivariate logistic regression analysis showed that the CTNNBIP1 rs935072 A>T SNP was an independent predictor for treatment response of EOC patients. Results of the genotype-phenotype correlation analysis further demonstrated that the rs935072 $\mathrm{T}$ allele was associated with increased mRNA expression levels of CTNNBIP1, a potential molecular marker for EOC patients to overcome chemoresistance. 
A 3'UTR of CTNNBIP1

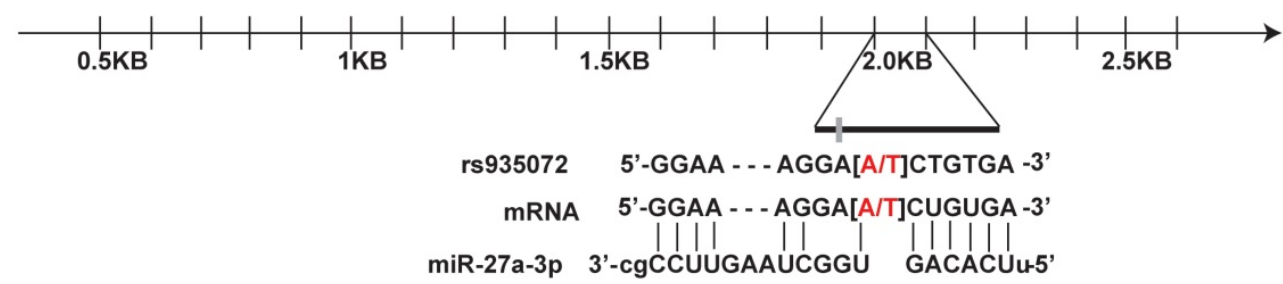

B

SV40 early enhancer

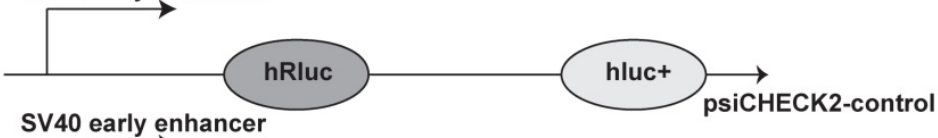

SV40 early enhancer

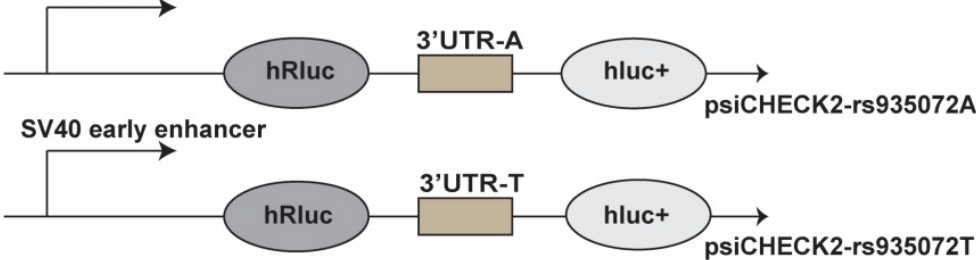

C
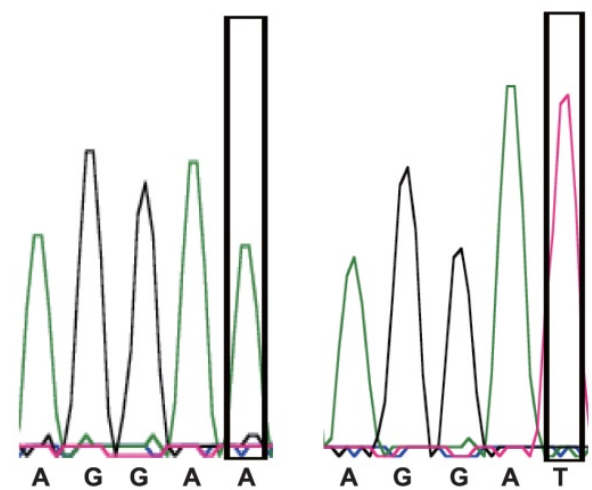

E

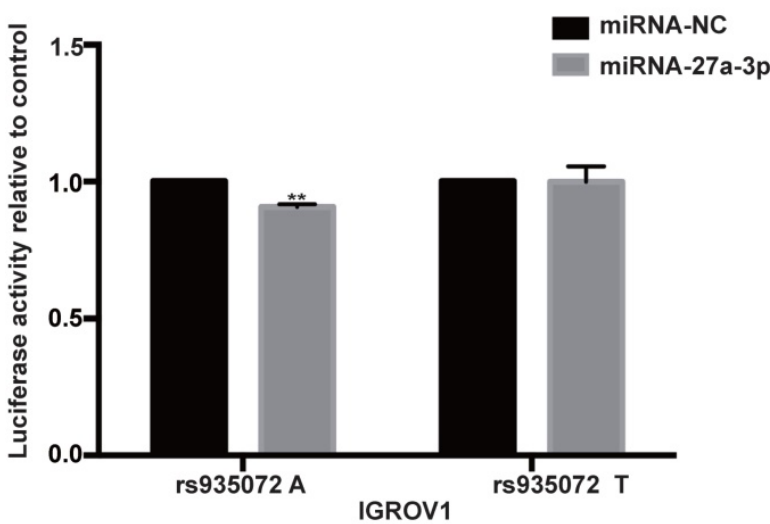

G

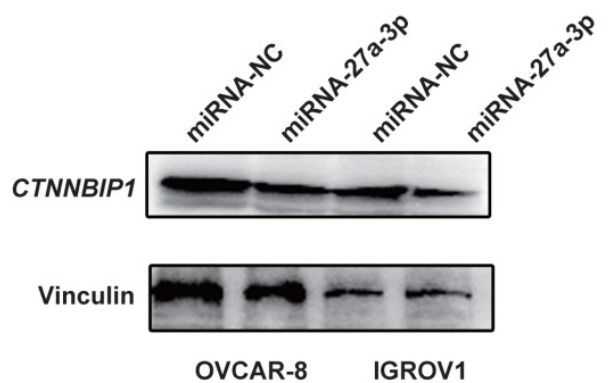

OVCAR-8 IGROV1

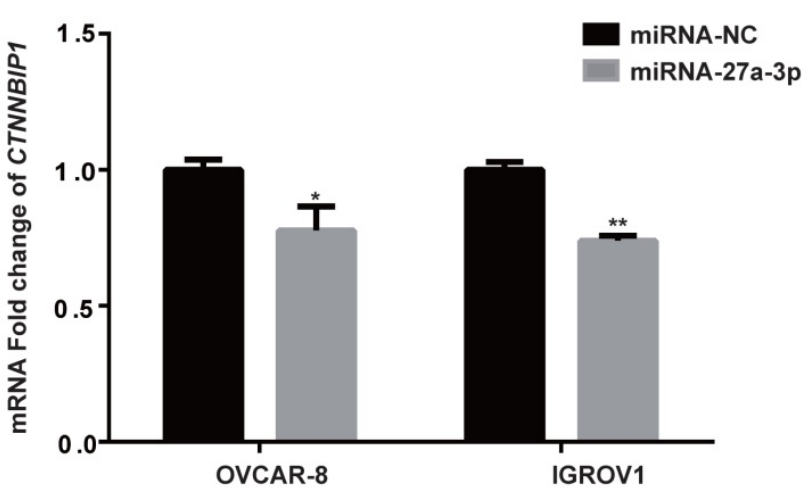

miRNA-NC

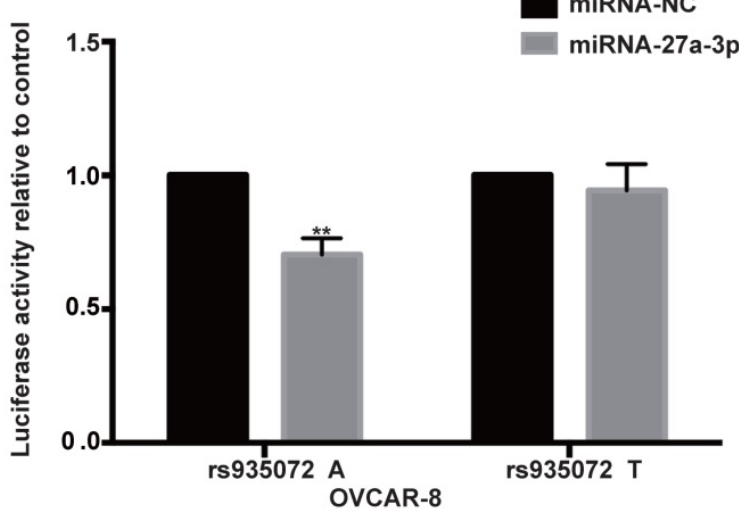

$\mathbf{F}$

Figure 3. The CTNNBIPI rs935072 A>T contributes to the decreased binding affinity of miR-27a-3p to the CTNNBIPI 3'UTR and increased the expression of CTNNBIPI. A, Graphic representation of the detailed location of rs 935072 in the 3'UTR of CTNNBIPI, which is also at the miRNA-binding site with the A allele. B, Schematic drawing of the luciferase reporter system. C. Sequencing results of the Psi-CHECK2 vector containing rs935072 A or T allele. Abbreviation: Mut, Mutagenesis. D-E, Luciferase activity in the presence of the miR-27a-3p transfected into IGROVI and OVACR-8 cell lines. F, The expression of CTNNBIPI was detected by the qRT-PCR assay in IGROVI and OVACR-8 cells overexpressing miR-27a-3p and control cells. G, The expression of CTNNBIPI was detected by western blot assay in IGROVI and OVACR-8 cells overexpressing miR-27a-3p and control cells. $* P<0.05$. $* * p<0.01$. 
A

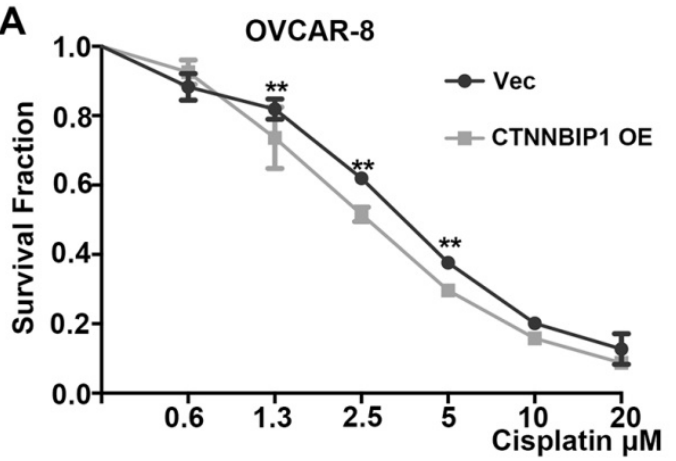

C

OVCAR-8
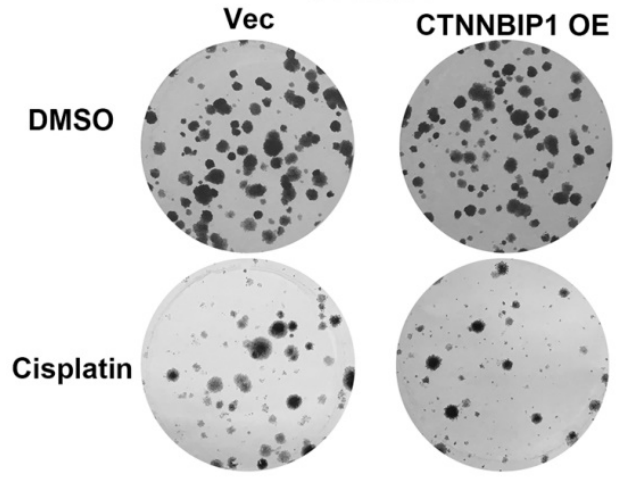

CTNNBIP1
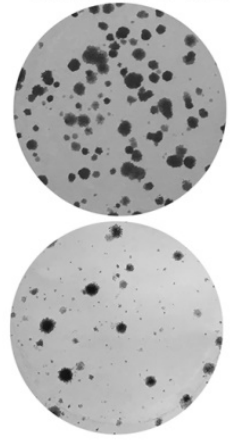

D

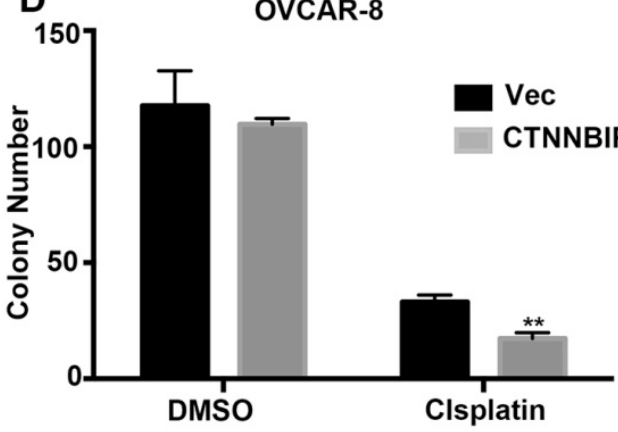

E

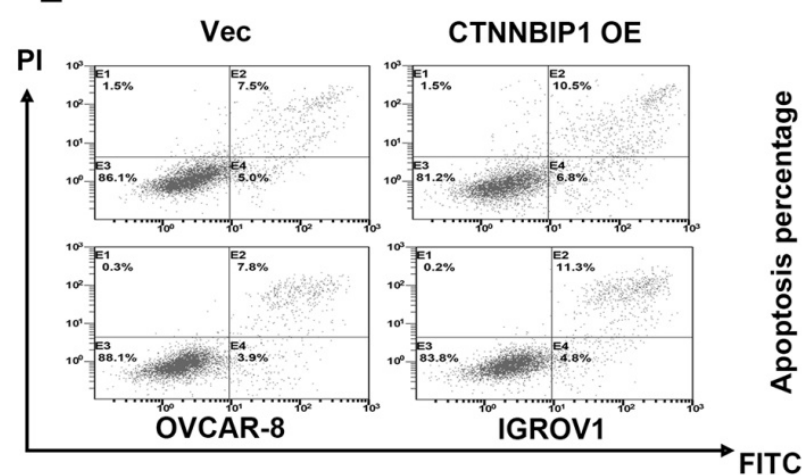

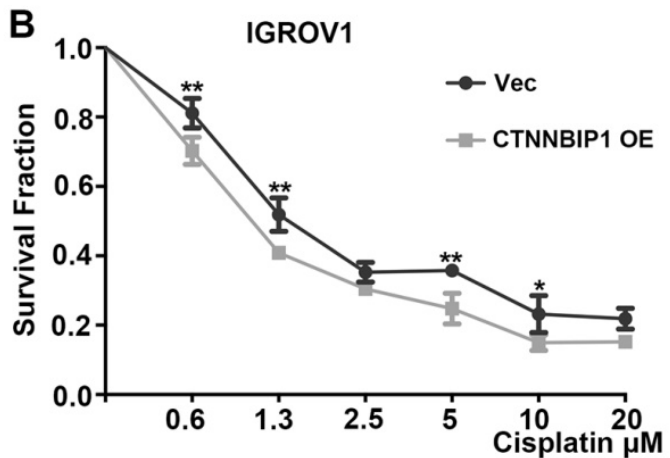

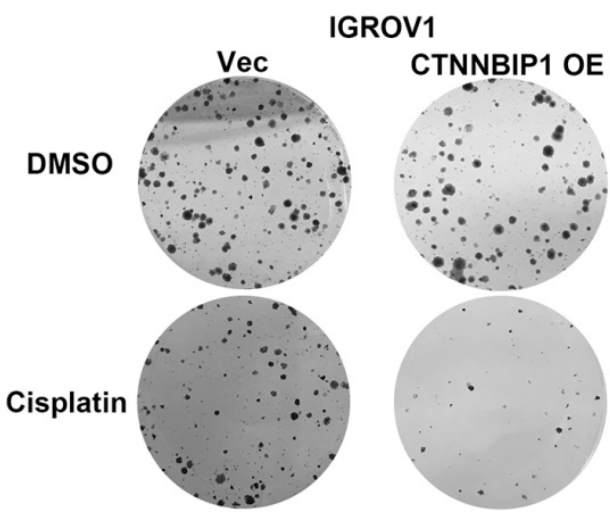

IGROV1

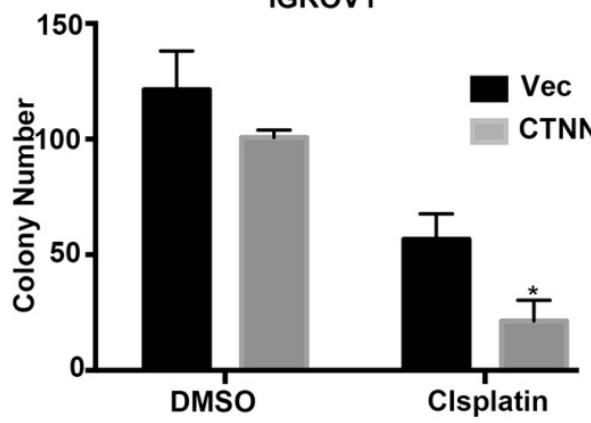

Apotosis

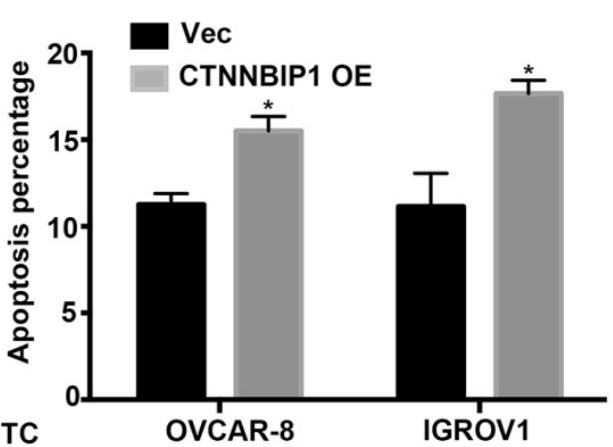

Figure 4. CTNNBIPI sensitizes ovarian cancer cells to cisplatin. A-B, Cell Counting Kit-8 assays showed the effect of empty vector and CTNNBIPI overexpression on the chemosensitivity of ovarian cancer cells to the cytotoxic effect of cisplatin. C-D, Colony formation assays showed the effect of empty vector and CTNNBIPI overexpression on the chemosensitivity of ovarian cancer cells to the cytotoxic effect of cisplatin. E, The percentage of apoptotic cells in indicated group after cisplatin treatment. Cells were stained with annexin $\mathrm{V}$-fluorescein isothiocyanate ( $\mathrm{FITC}$ ) and propidium iodide (PI) to detect cells in early apoptosis (annexin $\mathrm{V}+\mathrm{PI}-$ ) and late apoptosis (annexin $\mathrm{V}+\mathrm{PI}+$ ). Representative pictures are shown. $* P<0.05$. $* * P<0.01$.

The Wnt/ $\beta$-catenin signaling is an evolutionarily conserved and versatile pathway that is associated with many cellular processes and a wide variety of human diseases, especially for cancer [31]. $\beta$-Catenin serves as a core component in the Wnt/ $\beta$-catenin signaling. The stabilization of $\beta$-catenin is critical in cancer stem cell survival [32] and chemoresistance [33]. The $\beta$-catenin tends to accumulate in the 
cytoplasm and then is translocated into the nucleus, when aberrant WNT activation and mutations of the WNT gene or destruction of the complex components occurred [34]. Consequently, $\beta$-catenin binds to TCF/LEF and some other co-regulators to promote the transcription of target genes such as $c-M y c$ [35] and cyclin D1 [36], which initiate tumorigenesis. There are many negative regulation mechanisms of $\beta$-catenin. CTNNBIP1 is one of the molecules that inhibit $\beta$-catenin from forming a complex with TCF/ LEF and then inactivates the transcription of Wnt target genes, thereby negatively regulating the Wnt/ $\beta$-catenin pathway [15]. Here, we reported a novel regulatory mechanism of CTNNBIP1 activation, thereby uncovering a potential chemotherapeutic target for ovarian cancer treatment.

To date, few studies have reported the role of genetic variants in regulating the expression of CTNNBIP1, although rare somatic mutations in CTNNBIP1 were observed in some studies of malignancies [37, 38]. For example, in breast cancer, overall alterations (52-55\%) with frequent methylation (44-45\%) and deletion (20-32\%) of CTNNBIP1 were observed [39]. However, no studies explored the role of CTNNBIP1 SNPs in treatment response in ovarian cancer patients. To the best of our knowledge, the present study is the first that investigated associations between SNPs of CTNNBIP1 and the response to platinum treatment of Chinese EOC patients with functional validation, which provides an additional evidence to support the role of CTNNBIP1 rs935072 in predicting platinum-treatment response of Chinese EOC patients.

SNPs located at the gene 3'UTRs possibly affect cancer development and progression via regulating the efficiency of miRNA binding to the specific sites $[40,41]$. Previous studies demonstrated that miR-214 [42] and miR-603 [43] negatively regulated the expression levels of CTNNBIP1. In the present study, by using in silico tools, we identified miR-27a-3p as a new potential regulator of CTNNBIP1 expression. Since CTNNBIP1 rs935072 appears to be at a miR$27 a-3 p$ binding site, we observed a down-regulated luciferase activity for the rs 935072 A allele, compared with that for the $\mathrm{T}$ allele in both IGROV1 and OVCAR-8 cell lines. The observed decrease in luciferase activities meant an increased binding capacity of miR-27a-3p, thereby affecting the expression of CTNNBIP1. Furthermore, from the TCGA and Oncomine databases, we found that CTNNBIP1 might act as a chemosensitivity-related gene due to its relatively higher expression levels in chemosensitive ovarian cancer serious histology, compared with other types of histology. Further functional experiments confirmed that the overexpression of CTNNBIP1 sensitized ovarian cancer cell to cisplatin treatment. These results showed that the CTNNBIP1 rs935072 $\mathrm{T}$ allele may account for a good platinum response of EOC patients by modulating its mRNA expression.

Our findings may help identify subgroups of patients at a higher risk of developing chemoresistance and those patients who are more likely to benefit from individualized treatment strategies. However, the present study had several limitations. First of all, the inherent limitation was the retrospective study design with inevitable selection bias. Secondly, the sample size of study population was relatively small, which had limited statistical power to detect the true weak effect of SNPs on chemotherapeutic response in the analysis. Moreover, patients enrolled in the present study were from single cancer center and thus could not represent the general EOC patient population in China. Future multi-center validation is needed to substantiate our findings. Finally, additional in vivo studies are required to confirm our observed mechanism of CTNNBIP1 in platinum treatment response of ovarian cancer.

In conclusion, in the present study, we identified CTNNBIP1 rs935072 to be a potential biomarker for predicting platinum chemotherapeutic response for EOC patients. The underlying mechanism for the effect of the CTNNBIP1 rs935072 A>T change on chemotherapy treatment response resulted from a lower binding affinity of miR-27a-3p, thereby leading to the up-regulation of the CTNNBIP1 expression. Further in vitro experiments demonstrated that overexpression of CTNNBIP1 sensitized ovarian cancer cells to platinum treatment. Once further validated by other investigators, these findings would provide novel clues for individualized therapy for EOC patients in the future.

\section{Abbreviations}

EOC: epithelial ovarian cancer; SNPs: single nucleotide polymorphisms; OR: odds ratio; CI: confidence interval; GWASs: genome-wide association studies; CTNNBIP1: Catenin beta interacting protein 1; TCF/LEF: T-cell factor/ lymphoid enhancer factor; FUSCC: Fudan University Shanghai Cancer Center; SOCS: Shanghai Ovarian Cancer Study; FIGO: International Federation of Gynecology and Obstetrics; FDR: False Discovery rate; FPRP: false-positive report probability; BFDP: Bayesian false-discovery probability; LD: linkage disequilibrium; ROC: Receiver operating characteristic; AUC: area under the curve; DMEM: Dulbecco's modified Eagle's medium; SD: standard deviation. 


\section{Supplementary Material}

Supplementary figures and tables.

http://www.jcancer.org/v11p6850s1.pdf

\section{Acknowledgments}

This study was supported by Shanghai Natural Science Foundation (20ZR1412800) for Mengyun Wang, National Natural Science Foundation of China for Kexin Chen (Grant/Award Number: 81320108022), program for Changjiang Scholars and Innovative Research Team in University in China for Kexin Chen (Grant/Award Number: IRT_14R40) and Tianjin Science and Technology Committee Foundation for Kexin Chen (GrantNumber: 18YFZCSY00520).

\section{Competing Interests}

The authors have declared that no competing interest exists.

\section{References}

1. Jones OP, Melling JD, Ghaneh P. Adjuvant therapy in pancreatic cancer. World J Gastroenterol. 2014; 20: 14733-46.

2. Mari A, D'Andrea D, Abufaraj M, Foerster B, Kimura S, Shariat SF. Genetic determinants for chemo- and radiotherapy resistance in bladder cancer. Transl Androl Urol. 2017; 6: 1081-9.

3. Agarwal R, Kaye SB. Ovarian cancer: strategies for overcoming resistance to chemotherapy. Nat Rev Cancer. 2003; 3: 502-16.

4. Matsuo K, Eno ML, Im DD, Rosenshein NB, Sood AK. Clinical relevance of extent of extreme drug resistance in epithelial ovarian carcinoma. Gynecol Oncol. 2010; 116: 61-5

5. Kigawa J. New strategy for overcoming resistance to chemotherapy of ovarian cancer. Yonago Acta Med. 2013; 56: 43-50.

6. Bowtell DD. The genesis and evolution of high-grade serous ovarian cancer. Nat Rev Cancer. 2010; 10: 803-8.

7. Yu KD, Huang AJ, Fan L, Li WF, Shao ZM. Genetic variants in oxidative stress-related genes predict chemoresistance in primary breast cancer: a prospective observational study and validation. Cancer Res. 2012; 72: 408-19.

8. Akiyama S. [Genetic alterations and chemoresistance]. Gan To Kagaku Ryoho. 2005; 32: 1895-901.

9. Nozawa T, Minami H, Sugiura S, Tsuji A, Tamai I. Role of organic anion transporter OATP1B1 (OATP-C) in hepatic uptake of irinotecan and its active metabolite, 7-ethyl-10-hydroxycamptothecin: in vitro evidence and effect of single nucleotide polymorphisms. Drug Metab Dispos. 2005; 33: 434-9.

10. Zhou W, Gurubhagavatula S, Liu G, Park S, Neuberg DS, Wain JC, et al. Excision repair cross-complementation group 1 polymorphism predicts overall survival in advanced non-small cell lung cancer patients treated with platinum-based chemotherapy. Clin Cancer Res. 2004; 10: 4939-43.

11. Bergamaschi D, Gasco M, Hiller L, Sullivan A, Syed N, Trigiante G, et al. p53 polymorphism influences response in cancer chemotherapy via modulation of p73-dependent apoptosis. Cancer Cell. 2003; 3: 387-402.

12. Kim HS, Kim MK, Chung HH, Kim JW, Park NH, Song YS, et al. Genetic polymorphisms affecting clinical outcomes in epithelial ovarian cancer patients treated with taxanes and platinum compounds: A Korean population-based study. Gynecologic Oncology. 2009; 113: 264-9.

13. Tang N, Lyu D, Zhang Y, Liu HP. Association between the ERCC1 polymorphism and platinum-based chemotherapy effectiveness in ovarian cancer: a meta-analysis. Bmc Womens Health. 2017; 17: 43.

14. Arend RC, Londono-Joshi AI, Straughn JM, Jr., Buchsbaum DJ. The Wnt/beta-catenin pathway in ovarian cancer: a review. Gynecol Oncol. 2013; 131: 772-9.

15. Mao J, Fan S, Ma W, Fan P, Wang B, Zhang J, et al. Roles of Wnt/beta-catenin signaling in the gastric cancer stem cells proliferation and salinomycin treatment. Cell Death Dis. 2014; 5: e1039.

16. Sekiya $T$, Nakamura $T$, Kazuki $\mathrm{Y}$, Oshimura $\mathrm{M}$, Kohu $\mathrm{K}$, Tago $\mathrm{K}$, et al. Overexpression of Icat induces $\mathrm{G}(2)$ arrest and cell death in tumor cell mutants for adenomatous polyposis coli, beta-catenin, or Axin. Cancer Res. 2002; 62: 3322-6.

17. Mukerjee A, Ranjan AP, Vishwanatha JK. Targeted Nanocurcumin Therapy Using Annexin A2 Anitbody Improves Tumor Accumulation and Therapeutic Efficacy Against Highly Metastatic Breast Cancer. J Biomed Nanotechnol. 2016; 12: 1374-92.
18. Jiang $Y$, Ren W, Wang W, Xia J, Gou L, Liu M, et al. Inhibitor of beta-catenin and TCF (ICAT) promotes cervical cancer growth and metastasis by disrupting E-cadherin/beta-catenin complex. Oncology reports. 2017; 38: 2597-606.

19. Kosari-Monfared M, Nikbakhsh N, Fattahi S, Ghadami E, Ranaei M, Taheri H, et al. CTNNBIP1 downregulation is associated with tumor grade and viral infections in gastric adenocarcinoma. Journal of cellular physiology. 2019; 234: 2895-904.

20. Chen K, Ma H, Li L, Zang R, Wang C, Song F, et al. Genome-wide association study identifies new susceptibility loci for epithelial ovarian cancer in Han Chinese women. Nature communications. 2014; 5: 4682

21. Benjamini Y, Hochberg Y. Controlling the False Discovery Rate - a Practical and Powerful Approach to Multiple Testing. J R Stat Soc B. 1995; 57: 289-300.

22. Wakefield J. A Bayesian measure of the probability of false discovery in genetic epidemiology studies. Am J Hum Genet. 2007; 81: 208-27.

23. Li H, Tong $\mathrm{X}, \mathrm{Xu} \mathrm{Y}$, Wang M, Dai H, Shi T, et al. Functional genetic variants of RUVBL1 predict overall survival of Chinese patients with epithelial ovarian cancer. Carcinogenesis. 2019; 40: 1209-19.

24. Aulchenko YS, Ripke S, Isaacs A, van Duijn CM. GenABEL: an R library for genome-wide association analysis. Bioinformatics. 2007; 23: 1294-6.

25. Barrett JC, Fry B, Maller J, Daly MJ. Haploview: analysis and visualization of LD and haplotype maps. Bioinformatics. 2005; 21: 263-5.

26. Purcell S, Neale B, Todd-Brown K, Thomas L, Ferreira MA, Bender D, et al. PLINK: a tool set for whole-genome association and population-based linkage analyses. American journal of human genetics. 2007; 81: 559-75.

27. Li H, Lu Y, Pang Y, Li M, Cheng X, Chen J. Propofol enhances the cisplatin-induced apoptosis on cervical cancer cells via EGFR/JAK2/STAT3 pathway. Biomed Pharmacother. 2017; 86: 324-33.

28. Purcell S, Neale B, Todd-Brown K, Thomas L, Ferreira MAR, Bender D, et al. PLINK: A tool set for whole-genome association and population-based linkage analyses. Am J Hum Genet. 2007; 81: 559-75.

29. Winter WE, 3rd, Maxwell GL, Tian C, Carlson JW, Ozols RF, Rose PG, et al. Prognostic factors for stage III epithelial ovarian cancer: a Gynecologic Oncology Group Study. J Clin Oncol. 2007; 25: 3621-7.

30. Timmers PJ, Zwinderman AH, Teodorovic I, Vergote I, Trimbos JB. Clear cell carcinoma compared to serous carcinoma in early ovarian cancer: same prognosis in a large randomized trial. Int J Gynecol Cancer. 2009; 19: 88-93.

31. Clevers H, Nusse R. Wnt/beta-catenin signaling and disease. Cell. 2012; 149: 1192-205.

32. Condello S, Morgan CA, Nagdas S, Cao L, Turek J, Hurley TD, et al. beta-Catenin-regulated ALDH1A1 is a target in ovarian cancer spheroids. Oncogene. 2015; 34: 2297-308

33. Nagaraj AB, Joseph P, Kovalenko O, Singh S, Armstrong A, Redline R, et al. Critical role of Wnt/beta-catenin signaling in driving epithelial ovarian cancer platinum resistance. Oncotarget. 2015; 6: 23720-34.

34. White BD, Chien AJ, Dawson DW. Dysregulation of Wnt/beta-catenin signaling in gastrointestinal cancers. Gastroenterology. 2012; 142: 219-32.

35. Gekas C, D'Altri T, Aligue R, Gonzalez J, Espinosa L, Bigas A. beta-Catenin is required for T-cell leukemia initiation and MYC transcription downstream of Notch1. Leukemia. 2016; 30: 2002-10.

36. Tetsu $\mathrm{O}, \mathrm{McC}$ cormick F. beta-catenin regulates expression of cyclin D1 in colon carcinoma cells. Nature $1999 ; 398: 422-6$

37. Reifenberger J, Knobbe CB, Wolter M, Blaschke B, Schulte KW, Pietsch T, et al. Molecular genetic analysis of malignant melanomas for aberrations of the Wnt signaling pathway genes CTNNB1, APC, ICAT and BTRC. International Journal of Cancer. 2002; 100: 549-56.

38. Koyama T, Tago K, Nakamura T, Ohwada S, Morishita Y, Yokota J, et al. Mutation and expression of the beta-catenin-interacting protein ICAT in human colorectal tumors. Jpn J Clin Oncol. 2002; 32: 358-62.

39. Mukherjee N, Dasgupta H, Bhattacharya R, Pal D, Roy R, Islam S, et al. Frequent inactivation of MCC/CTNNBIP1 and overexpression of phospho-beta-catenin(Y654) are associated with breast carcinoma: Clinical and prognostic significance. Biochim Biophys Acta. 2016; 1862: 1472-84.

40. Yu Z, Li Z, Jolicoeur N, Zhang L, Fortin Y, Wang E, et al. Aberrant allele frequencies of the SNPs located in microRNA target sites are potentially associated with human cancers. Nucleic Acids Res. 2007; 35: 4535-41.

41. Saunders MA, Liang $\mathrm{H}, \mathrm{Li}$ WH. Human polymorphism at microRNAs and microRNA target sites. P Natl Acad Sci USA. 2007; 104: 3300-5.

42. Qi W, Chen JY, Cheng XM, Huang JN, Xiang T, Li QJ, et al. Targeting the Wnt-Regulatory Protein CTNNBIP1 by microRNA-214 Enhances the Stemness and Self-Renewal of Cancer Stem-Like Cells in Lung Adenocarcinomas. Stem Cells. 2015; 33: 3423-36.

43. Guo M, Zhang XM, Wang GZ, Sun JH, Jiang ZF, Khadarian K, et al. miR-603 promotes glioma cell growth via Wnt/beta-catenin pathway by inhibiting WIF1 and CTNNBIP1. Cancer Letters. 2015; 360: 76-86. 